\title{
CRISPR-Cas12a target binding unleashes single-stranded DNase activity
}

Janice S. Chen ${ }^{1} \dagger$, Enbo $\mathrm{Ma}^{1} \dagger$, Lucas B. Harrington ${ }^{1} \dagger$, Xinran $\operatorname{Tian}^{2}$ and Jennifer A. Doudna $a^{1-5 *}$

\author{
Affiliations: \\ ${ }^{1}$ Department of Molecular and Cell Biology \\ ${ }^{2}$ Department of Chemistry \\ ${ }^{3}$ Innovative Genomics Institute \\ ${ }^{4}$ Howard Hughes Medical Institute \\ University of California, Berkeley, Berkeley, California 94720, USA. \\ ${ }^{5}$ Molecular Biophysics \& Integrated Bioimaging Division, Lawrence Berkeley National \\ Laboratory, Berkeley, CA 94720, USA. \\ *Correspondence to: $\underline{\text { doudna@berkeley.edu }}$ \\ $\dagger$ These authors contributed equally to this work.
}


Abstract: CRISPR-Cas12a (Cpf1) proteins are RNA-guided DNA targeting enzymes that bind and cut DNA as components of bacterial adaptive immune systems. Like CRISPR-Cas9, Cas12a can be used as a powerful genome editing tool based on its ability to induce genetic changes in cells at sites of double-stranded DNA (dsDNA) cuts. Here we show that RNA-guided DNA binding unleashes robust, non-specific single-stranded DNA (ssDNA) cleavage activity in Cas12a sufficient to completely degrade both linear and circular ssDNA molecules within minutes. This activity, catalyzed by the same active site responsible for site-specific dsDNA cutting, indiscriminately shreds ssDNA with rapid multiple-turnover cleavage kinetics. Activation of ssDNA cutting requires faithful recognition of a DNA target sequence matching the 20-nucleotide guide RNA sequence with specificity sufficient to distinguish between closely related viral serotypes. We find that target-dependent ssDNA degradation, not observed for CRISPR-Cas9 enzymes, is a fundamental property of type V CRISPR-Cas12 proteins, revealing a fascinating parallel with the RNA-triggered general RNase activity of the type VI CRISPRCas13 enzymes.

One Sentence Summary: Cas12a (Cpfl) and related type V CRISPR interference proteins possess non-specific, single-stranded DNase activity upon activation by guide RNA-dependent DNA binding. 


\section{Main Text:}

CRISPR-Cas adaptive immunity in bacteria and archaea uses RNA-guided nucleases to target and degrade foreign nucleic acids $(1,2)$. The CRISPR-Cas9 family of proteins has been widely deployed for gene editing applications $(3,4)$ based on the precision of double-stranded DNA (dsDNA) cleavage induced by two catalytic domains, RuvC and $\mathrm{HNH}$, at sequences complementary to a guide RNA sequence $(5,6)$. A second family of enzymes, CRISPR-Cas12a (Cpf1), uses a single RuvC catalytic domain for guide RNA-directed dsDNA cleavage (7-12)

(Fig. 1a). Distinct from Cas9, Cas12a enzymes recognize a T-rich protospacer adjacent motif (PAM) (7), catalyze their own guide RNA (crRNA) maturation (13) and generate a PAM-distal dsDNA break with staggered 5' and 3' ends (7), features that have attracted interest for gene editing applications (14-10). However, the substrate specificity and DNA cleavage mechanism of Cas12a are yet to be fully elucidated.

While investigating substrate requirements for Cas12a activation, we tested Lachnospiraceae bacterium ND2006 Cas12a (LbCas12a) for guide RNA-directed singlestranded DNA (ssDNA) cleavage, a capability of diverse CRISPR-Cas9 orthologs $(17,18)$. Purified LbCas12a or Streptococcus pyogenes Cas9 (SpCas9) proteins (fig. S1) were assembled with guide RNA sequences targeting a circular, single-stranded M13 DNA phage. In contrast to SpCas9, we were surprised to find that LbCas12a induced rapid and complete degradation of M13 by a cleavage mechanism that could not be explained by sequence-specific DNA cutting (Fig. 1b). This ssDNA shredding activity, not observed using an LbCas 12a protein containing inactivating mutations in the RuvC catalytic domain, raised the possibility that a target-bound LbCas 12 a could degrade any ssDNA, regardless of complementarity to the guide RNA. To test this idea, we assembled LbCas12a or SpCas9 with a different guide RNA and complementary ssDNA with no sequence homology to M13 phage genome sequence, and added M13 DNA to 
the reaction. Remarkably, LbCas12a catalyzed M13 degradation only in the presence of this complementary ssDNA “activator”, an activity not observed for SpCas9 (Fig. 1c). These findings reveal that binding of LbCas12a to a guide-complementary ssDNA unleashes robust, non-specific ssDNA trans-cleavage activity.

We next investigated the requirements for LbCas12-catalyzed trans-cleavage activity. Using a fluorophore quencher (FQ)-labeled reporter assay $(19,20)$, we assembled LbCas 12a with its crRNA and either a complementary ssDNA, dsDNA or single-stranded RNA (ssRNA), and introduced an unrelated ssDNA- or ssRNA-FQ reporter in trans (fig. S2). Both the crRNAcomplementary ssDNA or dsDNA (the activator) triggered LbCas12a to cleave the ssDNA-FQ reporter substrate (fig. S2A). However, ssRNA was neither capable of activating trans-cleavage nor susceptible to degradation by LbCas12a (fig. S2B), confirming that LbCas12a harbors a DNA-activated general DNase activity.

To determine how LbCas12a-catalyzed ssDNA cleavage activity relates to site-specific dsDNA cutting, we tested the length requirements of the target strand (TS) and non-target strand (NTS) for LbCas12a activation using radiolabeled oligonucleotides. Although TS cutting occurred irrespective of the NTS length (figs. S3A-B), NTS cleavage occurred only when the TS contained at least 15 nucleotides (nt) of complementarity with the crRNA (fig. S3C). This showed that TS recognition is a prerequisite for NTS cutting. To test whether LbCas 12a remains active for non-specific ssDNA cleavage after sequence-specific binding and cleavage of a dsDNA substrate, we first cut a dsDNA plasmid with an LbCas12a-crRNA complex, and introduced an unrelated dsDNA or ssDNA to the reaction (Fig. 2a). Whereas non-specific dsDNA substrate remained intact, the ssDNA was rapidly degraded in a RuvC-domain dependent manner (Fig. 2a; figs. S4, S5). Together, these results show that RNA-guided DNA 
binding activates LbCas12a for both site-specific dsDNA cutting and non-specific ssDNA transcleavage.

The rapid degradation of a trans substrate suggested that the kinetics of LbCas12acatalyzed site-specific dsDNA ( cis- $\left.^{-}\right)$cleavage and non-specific ssDNA (trans-) cleavage are fundamentally different. Stoichiometric titration experiments showed that cis-cleavage is singleturnover (21) (Fig. 2b), whereas trans-cleavage is multiple-turnover (Fig. 2c). Using the FQ assay, we found that LbCas12a-crRNA bound to a ssDNA activator molecule catalyzed ssDNA trans-cleavage at a rate of $\sim 250$ turnovers per second and a catalytic efficiency $\left(k_{\text {cat }} / K_{\mathrm{m}}\right)$ of $5.1 \times 10^{8} \mathrm{~s}^{-1} \mathrm{M}^{-1}$. When bound to a dsDNA activator, LbCas12a-crRNA catalyzed $\sim 1250$ turnovers per second with a catalytic efficiency approaching the rate of diffusion (22) with a $k_{\text {cat }} / K_{\mathrm{m}}$ of $1.7 \times 10^{9} \mathrm{~s}^{-1} \mathrm{M}^{-1}$ (Fig. 2d; fig. S6). These differences suggest that the NTS of the dsDNA activator helps stabilize the Cas12a complex in an optimal conformation for transssDNA cutting.

We next tested the specificity of trans-cleavage activation using either a ssDNA or dsDNA activator. We found that the PAM sequence required for dsDNA binding by CRISPRCas12a (21) is critical for catalytic activation by a crRNA-complementary dsDNA $(8,23)$, but not for a crRNA-complementary ssDNA (Fig. 3a). Two base-pair (bp) mismatches introduced along the crRNA-complementary sequence of either a ssDNA or dsDNA activator molecule slowed the trans-cleavage rate of a ssDNA-FQ reporter by up to $\sim 100$ fold, depending on the mismatch position. For only the dsDNA activator, alterations to the PAM sequence or mismatches between the crRNA and PAM-adjacent "seed region" also had large inhibitory effects on trans-ssDNA cleavage activity (Fig. 3b; fig. S7), similar to the mismatch tolerance pattern observed in Cas12a off-target studies (24-26). Together, these data are consistent with 
PAM-mediated dsDNA target binding and the role of base pairing between the crRNA and the target strand to activate trans-ssDNA cutting.

We next explored whether LbCas12a-catalyzed trans-ssDNA cleavage can distinguish between two closely-related dsDNA viruses, human papillomavirus (HPV) serotypes 16 (HPV16) and 18 (HPV18) (27). Within the HPV16 and HPV18 genomes, we selected a $20 \mathrm{nt}$ target sequence located next to a TTTA PAM that varied by only six base pairs between the two serotypes (Fig. 3c). Plasmids containing a 500 bp fragment of the HPV16 or HPV18 genome, including the target sequence, were incubated with the LbCas12a-crRNA complex targeting either the HPV16 or HPV18 fragment and a fluorescent ssDNA reporter. Robust LbCas12acatalyzed ssDNA trans-cleavage occurred only in the presence of the cognate HPV target (Fig. 3c; fig. S8), suggesting that dsDNA recognition specificity and trans-cleavage activity could in principle be extended to detect any dsDNA sequence.

We wondered if this trans-ssDNA cutting activity might be a property of Cas $12 \mathrm{a}$, and perhaps more evolutionarily distinct type V CRISPR effector proteins, considering that all type V effectors contain a single RuvC nuclease domain $(28,29)$. We therefore purified Cas 12a orthologs from Acidaminococcus sp. (AsCas12a) and Francisella novicida (FnCas12a), as well as a Cas12b protein from Alicyclobacillus acidoterrestris (AaCas12b) to test for cis- and transssDNA cleavage activity. With varying efficiencies, all of these homologs catalyzed non-specific ssDNase cleavage when assembled with crRNA and a complementary ssDNA activator (Fig. 4a), suggesting that target-dependent activation of non-specific ssDNA cleavage is a fundamental feature of all type V CRISPR-Cas12-family proteins.

Together, these findings support a unifying mechanism of target interference that begins with the Cas12a-guide RNA complex binding to a complementary DNA sequence in a PAM- 
dependent (dsDNA) or PAM-independent (ssDNA) manner (Fig. 4b). Within a host bacterium, such enzyme activation could provide simultaneous protection from both dsDNA and ssDNA phages, and could also target ssDNA sequences that arise temporarily during phage replication or transcription (30). For genome editing applications, this newly identified activity may also degrade single-stranded oligonucleotide donors that are often used for homology directed repair (31), altering DNA repair outcomes. Finally, these results reveal unexpected functional convergence of target-dependent trans-cleavage activity between the DNA-targeting type V and RNA-targeting type VI CRISPR effector enzymes $(19,32,33)$ and broaden the antiviral capabilities of CRISPR interference. 


\section{References:}

1. R. Sorek, V. Kunin, P. Hugenholtz, CRISPR--a widespread system that provides acquired resistance against phages in bacteria and archaea. Nat Rev Microbiol 6, 181-186 (2008).

2. K. S. Makarova et al., An updated evolutionary classification of CRISPR-Cas systems. Nat Rev Microbiol 13, 722-736 (2015).

3. J. A. Doudna, E. Charpentier, Genome editing. The new frontier of genome engineering with CRISPR-Cas9. Science 346, 1258096 (2014).

4. R. Barrangou, P. Horvath, A decade of discovery: CRISPR functions and applications. Nat Microbiol 2, 17092 (2017).

5. M. Jinek et al., A programmable dual-RNA-guided DNA endonuclease in adaptive bacterial immunity. Science 337, 816-821 (2012).

6. J. S. Chen, J. A. Doudna, The chemistry of Cas9 and its CRISPR colleagues. Nat Rev Chem 1, (2017).

7. B. Zetsche et al., Cpf1 is a single RNA-guided endonuclease of a class 2 CRISPR-Cas system. Cell 163, 759-771 (2015).

8. D. C. Swarts, J. van der Oost, M. Jinek, Structural Basis for Guide RNA Processing and Seed-Dependent DNA Targeting by CRISPR-Cas12a. Mol Cell 66, 221-233 e224 (2017).

9. D. Dong et al., The crystal structure of Cpf1 in complex with CRISPR RNA. Nature 532, $522-526(2016)$.

10. P. Gao, H. Yang, K. R. Rajashankar, Z. Huang, D. J. Patel, Type V CRISPR-Cas Cpf1 endonuclease employs a unique mechanism for crRNA-mediated target DNA recognition. Cell Res 26, 901-913 (2016). 
11. S. Stella, P. Alcon, G. Montoya, Structure of the Cpf1 endonuclease R-loop complex after target DNA cleavage. Nature 546, 559-563 (2017).

12. T. Yamano et al., Crystal Structure of Cpf1 in Complex with Guide RNA and Target DNA. Cell 165, 949-962 (2016).

13. I. Fonfara, H. Richter, M. Bratovic, A. Le Rhun, E. Charpentier, The CRISPR-associated DNA-cleaving enzyme Cpf1 also processes precursor CRISPR RNA. Nature 532, 517$521(2016)$.

14. B. Zetsche et al., Multiplex gene editing by CRISPR-Cpf1 using a single crRNA array. Nat Biotechnol 35, 31-34 (2017).

15. X. Tang et al., A CRISPR-Cpf1 system for efficient genome editing and transcriptional repression in plants. Nat Plants 3, 17018 (2017).

16. Y. E. Tak et al., Inducible and multiplex gene regulation using CRISPR-Cpf1-based transcription factors. Nat Methods, (2017).

17. Y. Zhang, R. Rajan, H. S. Seifert, A. Mondragon, E. J. Sontheimer, DNase H Activity of Neisseria meningitidis Cas9. Mol Cell 60, 242-255 (2015).

18. E. Ma, L. B. Harrington, M. R. O'Connell, K. Zhou, J. A. Doudna, Single-Stranded DNA Cleavage by Divergent CRISPR-Cas9 Enzymes. Mol Cell 60, 398-407 (2015).

19. A. East-Seletsky et al., Two distinct RNase activities of CRISPR-C2c2 enable guideRNA processing and RNA detection. Nature 538, 270-273 (2016).

20. J. S. Gootenberg et al., Nucleic acid detection with CRISPR-Cas13a/C2c2. Science 356, 438-442 (2017).

21. D. Singh et al., Real-time observation of DNA target interrogation and product release by the RNA-guided endonuclease CRISPR Cpfl. bioRxiv, (2017). 
22. R. A. Alberty, G. G. Hammes, Application of the Theory of Diffusion-Controlled Reactions to Enzyme Kinetics. J Phys Chem-Us 62, 154-159 (1958).

23. S. H. Sternberg, S. Redding, M. Jinek, E. C. Greene, J. A. Doudna, DNA interrogation by the CRISPR RNA-guided endonuclease Cas9. Nature 507, 62-67 (2014).

24. D. Kim et al., Genome-wide analysis reveals specificities of Cpf1 endonucleases in human cells. Nat Biotechnol 34, 863-868 (2016).

25. B. P. Kleinstiver et al., Genome-wide specificities of CRISPR-Cas Cpf1 nucleases in human cells. Nat Biotechnol 34, 869-874 (2016).

26. H. K. Kim et al., In vivo high-throughput profiling of CRISPR-Cpf1 activity. Nat Methods 14, 153-159 (2017).

27. C. B. Woodman, S. I. Collins, L. S. Young, The natural history of cervical HPV infection: unresolved issues. Nat Rev Cancer 7, 11-22 (2007).

28. S. Shmakov et al., Diversity and evolution of class 2 CRISPR-Cas systems. Nat Rev Microbiol 15, 169-182 (2017).

29. E. V. Koonin, K. S. Makarova, F. Zhang, Diversity, classification and evolution of CRISPR-Cas systems. Curr Opin Microbiol 37, 67-78 (2017).

30. P. Samai et al., Co-transcriptional DNA and RNA Cleavage during Type III CRISPR-Cas Immunity. Cell 161, 1164-1174 (2015).

31. C. D. Richardson, G. J. Ray, M. A. DeWitt, G. L. Curie, J. E. Corn, Enhancing homology-directed genome editing by catalytically active and inactive CRISPR-Cas9 using asymmetric donor DNA. Nature Biotechnology 34, 339-+ (2016).

32. O. O. Abudayyeh et al., $\mathrm{C} 2 \mathrm{c} 2$ is a single-component programmable RNA-guided RNAtargeting CRISPR effector. Science 353, aaf5573 (2016). 
33. A. A. Smargon et al., Cas13b Is a Type VI-B CRISPR-Associated RNA-Guided RNase Differentially Regulated by Accessory Proteins Csx27 and Csx28. Mol Cell 65, 618-630 e617 (2017)

Acknowledgments: We thank O. Mavrothalassitis, D. Burstein, D. Lee and members of the Doudna laboratory for comments and discussions. This research was supported in part by the Allen Distinguished Investigator Program, through The Paul G. Allen Frontiers Group, and the National Science Foundation (MCB-1244557 to J.A.D.). J.S.C. and L.B.H. are supported by National Science Foundation Graduate Research Fellowships. J.A.D. is an Investigator of the Howard Hughes Medical Institute and executive director of the Innovative Genomics Institute at the University of California, Berkeley and the University of California, San Francisco. J.A.D. is a co-founder of Editas Medicine, Intellia Therapeutics, and Caribou Biosciences and a scientific adviser to Caribou, Intellia, eFFECTOR Therapeutics and Driver. The Regents of the University of California have patents pending for CRISPR technologies on which the authors are inventors. 


\section{Figures:}

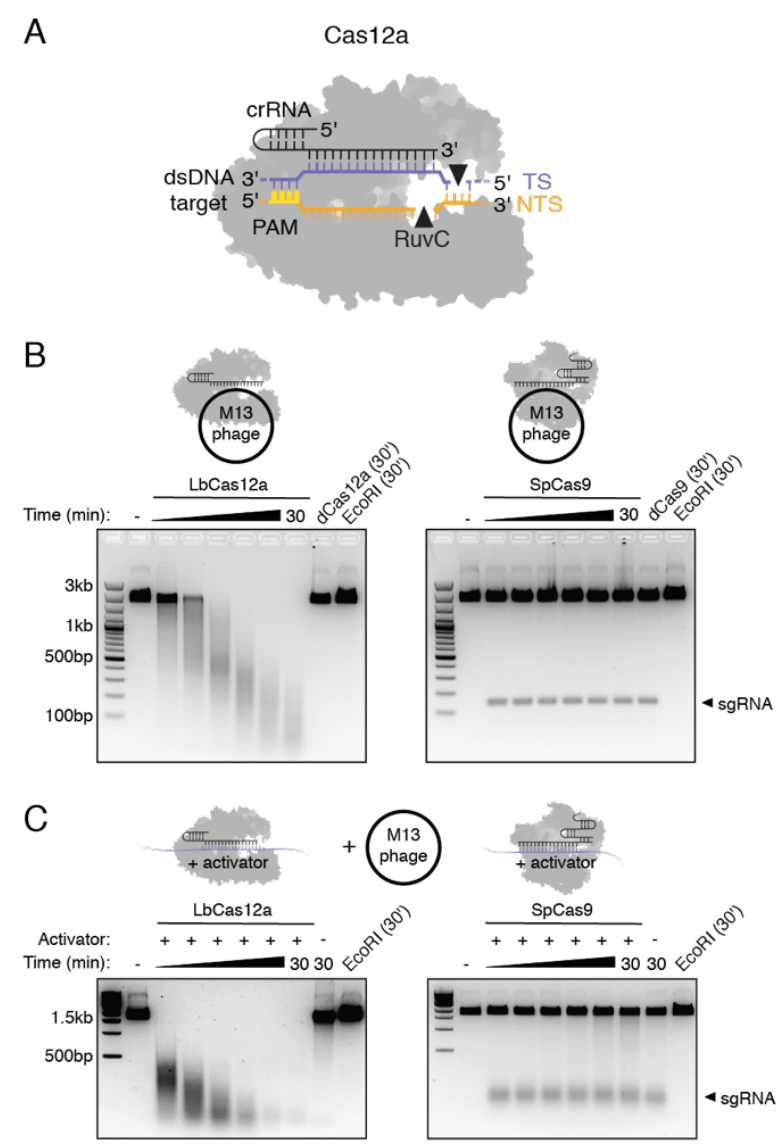

Fig. 1. Cas12a target recognition activates non-specific single-stranded DNA cleavage. (A)

Cas12a-crRNA complex binds a dsDNA substrate and generates a 5' overhang staggered cut using a single RuvC nuclease. (B, C) Representative M13 ssDNA cleavage timecourses with purified LbCas12a (left) and SpCas9 (right) complexed with a (B) guide RNA complementary to M13 phage or (C) a guide RNA and complementary ssDNA activator with no sequence homology to M13 phage. 

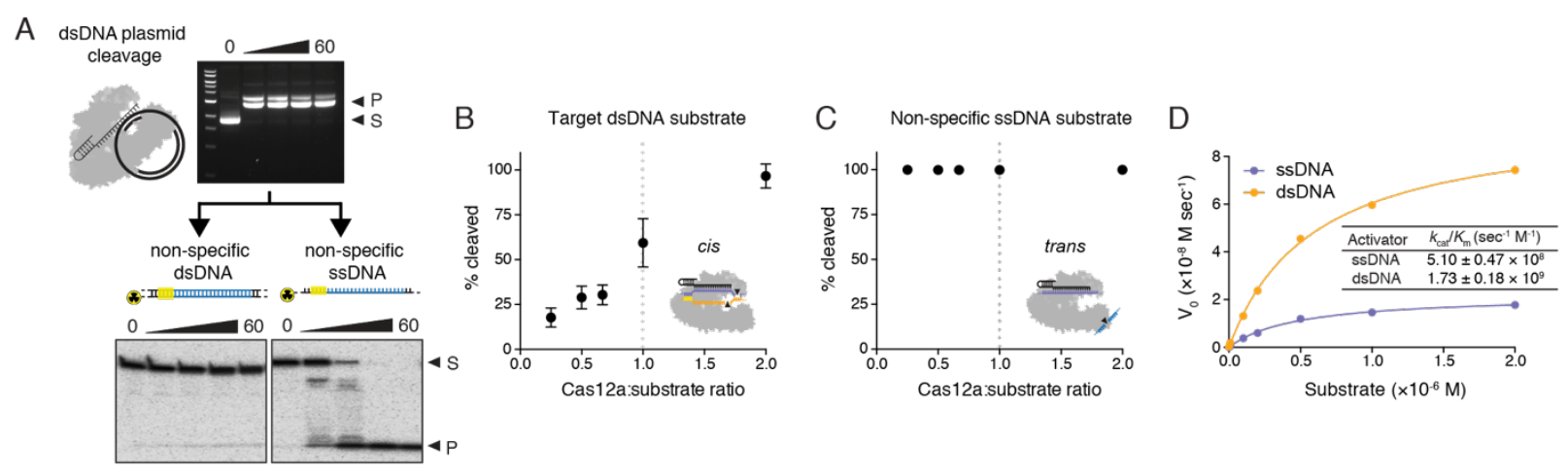

Fig. 2. Kinetics of Cas12a ssDNA trans-cleavage. (A) Sequence-specific plasmid DNA

cleavage reactions by LbCas12a-crRNA (top) were introduce to a separate radiolabeled dsDNA or ssDNA substrate of unrelated sequence (bottom); timecourses represent minutes. (B) Target dsDNA or (C) non-specific ssDNA incubated with molar ratios of LbCas12a-crRNA as indicated. Each point represents the mean quantified percent cleavage after 30 minutes at $37^{\circ} \mathrm{C}$, at which time the reaction was at completion. Error bars represent the mean \pm s.d., where $n=3$. (D) Representative Michaelis-Menten plot for LbCas12a-catalyzed ssDNA trans-cleavage using a dsDNA or ssDNA activator. Measured $k_{\text {cat }} / K_{\mathrm{m}}$ values report mean \pm s.d., where $n=3$. 

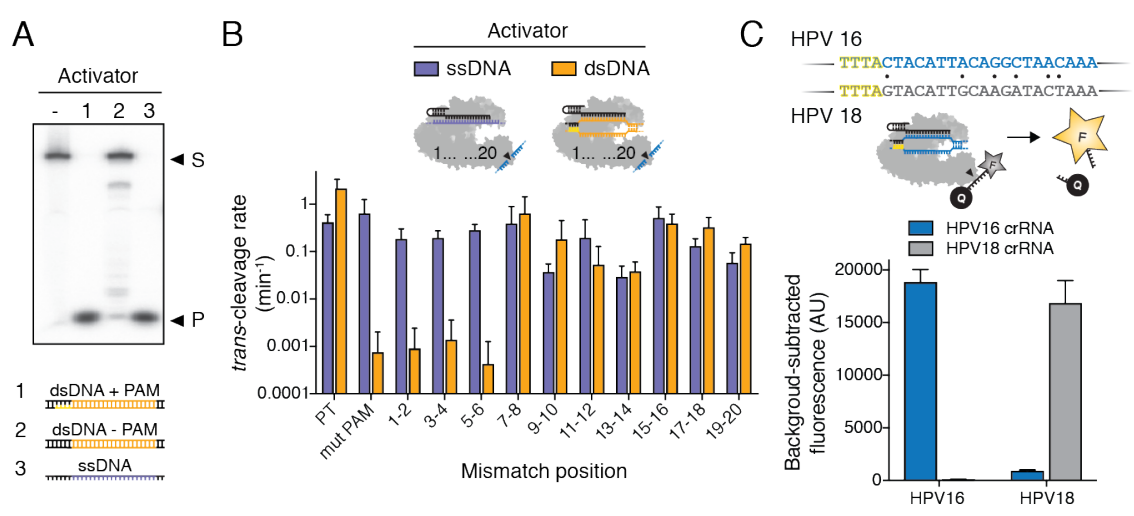

Fig. 3. Specificity of trans-cleavage activation. (A) LbCas12a-crRNA in the absence or presence of indicated activator, incubated with a radiolabeled non-specific ssDNA substrate (S) for $30 \mathrm{~min}$ at $37^{\circ} \mathrm{C}$; products $(\mathrm{P})$ resolved by denaturing PAGE. (B) Observed trans-cleavage rates for LbCas12a using a ssDNA or dsDNA activator with indicated mismatches; rates represent the average of three different targets measured in triplicate, and error bars represent mean \pm s.d., where $n=9$. (C) 20 base-pair target sequence with adjacent TTTA PAM (yellow) in related dsDNA human papillomavirus (HPV) serotypes 16 (HPV16) and 18 (HPV18) differs by six base pairs (black dots). Binding to a cognate HPV sequence activates LbCas12a transcleavage of the DNaseAlert substrate (see Methods) and generates a fluorescent signal. Background-subtracted maximum fluorescence values represent mean \pm s.d., where $n=3$. 


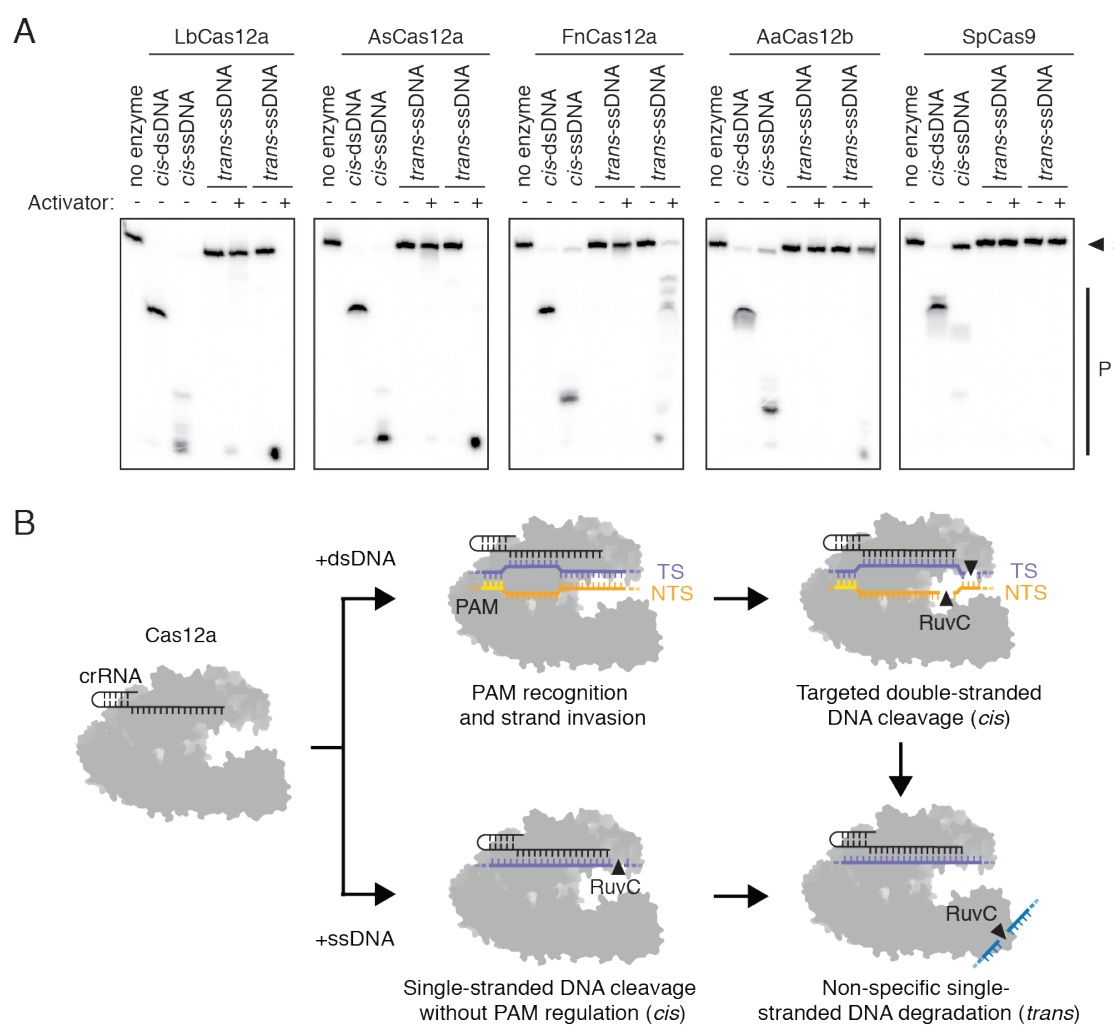

Fig. 4. Activator-dependent, non-specific ssDNA cleavage activity is conserved across type

V CRISPR systems. (A) Radiolabeled cis (complementary) or trans (non-complementary)

substrates were incubated with Cas12-crRNA or Cas9-sgRNA in the presence or absence of a ssDNA activator for $30 \mathrm{~min}$ at $37^{\circ} \mathrm{C}$ (or $47.5^{\circ} \mathrm{C}$ for AaCas $12 \mathrm{~b}$ ); substrate (S) and nucleotide products (P) resolved by denaturing PAGE. (B) Model for PAM-dependent and PAMindependent activation of $c$ is and trans-cleavage by Cas12a. 


\section{Materials and Methods}

Protein expression and purification. SpCas9 and Cas 12 proteins and mutants were cloned into a custom pET-based expression vector containing an N-terminal 10×His-tag, maltose-binding protein (MBP) and TEV protease cleavage site. Point mutations were introduced by around-thehorn PCR and verified by DNA sequencing. Proteins were purified as described (1), with the following modifications: E. coli BL21(DE3) containing SpCas9 or Cas12 expression plasmids were grown in Terrific Broth at $16^{\circ} \mathrm{C}$ for $14 \mathrm{hr}$. Cells were harvested and resuspended in Lysis Buffer (50 mM Tris-HCl, pH 7.5, 500 mM NaCl, 5\% (v/v) glycerol, 1 mM TCEP, 0.5 mM PMSF and $0.25 \mathrm{mg} / \mathrm{ml}$ lysozyme), disrupted by sonication, and purified using Ni-NTA resin. After overnight TEV cleavage at $4^{\circ} \mathrm{C}$, proteins were purified over an MBPTrap HP column connected to a HiTrap Heparin HP column for cation exchange chromatography. The final gel filtration step (Superdex 200) was carried out in elution buffer containing $20 \mathrm{mM}$ Tris-HCl, $\mathrm{pH}$ 7.5, $200 \mathrm{mM} \mathrm{NaCl}$ (or $250 \mathrm{mM} \mathrm{NaCl}$ for AaCas12b), 5\% (v/v) glycerol and $1 \mathrm{mM}$ TCEP. All proteins tested in this study are shown in fig. S1.

Nucleic acid preparation. DNA substrates were synthesized commercially (IDT). For FQreporter assays, activator DNA duplexes were prepared by annealing 5-fold molar excess of the NTS to TS in $1 \times$ hybridization buffer (20 nM Tris-Cl, $\mathrm{pH} 7.5,100 \mathrm{mM} \mathrm{KCl,} 5 \mathrm{mM} \mathrm{MgCl}$ ), heating at $95^{\circ} \mathrm{C}$ and slow-cooling on the benchtop. HPV16 and HPV18 fragments were synthesized as gBlocks (IDT) and cloned into a custom pET-based vector via Gibson assembly. For radiolabeled cleavage assays, PAGE-purified DNA oligos were prepared as described.

sgRNA templates were PCR amplified from a pUC19 vector or overlapping primers containing a T7 promoter, 20 nucleotide target sequence and an sgRNA scaffold. The amplified PCR product served as the DNA template for in vitro transcription reactions, which were 
performed as described (1). crRNAs were transcribed in vitro using a single-stranded DNA template containing a T7 promoter, repeat and spacer in the reverse complement orientation, which was annealed to T7 forward primer in $1 \times$ hybridization buffer. All DNA and RNA substrates are listed in Table $\mathbf{S 1 .}$

DNA cleavage assays. Generally, Cas $12 \mathrm{a}$-mediated cleavage assays were carried out in Cleavage Buffer consisting of $20 \mathrm{mM}$ HEPES (pH 7.5), $150 \mathrm{mM} \mathrm{KCl,} 10 \mathrm{mM} \mathrm{MgCl}_{2}, 1 \%$ glycerol and 0.5 mM DTT. For M13-targeting assays, $30 \mathrm{nM}$ Cas12a was pre-assembled with either $36 \mathrm{nM}$ of M13-targeting crRNA (cis) or with $36 \mathrm{nM}$ of crRNA and $40 \mathrm{nM}$ complementary ssDNA (activator) with no sequence homology to $\mathrm{M} 13$ (trans) at $37^{\circ} \mathrm{C}$ for $10 \mathrm{~min}$. The reaction was initiated by adding $10 \mathrm{nM}$ M13mp18 ssDNA (New England Biolabs) and incubated at $37^{\circ} \mathrm{C}$ for indicated timepoints. Reactions were quenched with DNA loading buffer $(30 \%(\mathrm{v} / \mathrm{v})$ glycerol, $0.25 \%(\mathrm{w} / \mathrm{v})$ bromophenol blue, $0.25 \%(\mathrm{w} / \mathrm{v})$ xylene cyanol) containing $15 \mathrm{mM}$ EDTA and separated by $1.5 \%$ agarose gel pre-stained with SyberGold.

For radiolabeled cleavage assays, the substrates used were 5'-end-labeled with T4 PNK (NEB) in the presence of gamma ${ }^{32} \mathrm{P}-\mathrm{ATP}$. For dsDNA substrates, the non-target strand was first 5'-end-labeled and then annealed with excess corresponding target strand. The concentrations of Cas12a (or SpCas9), guide RNA and ${ }^{32} \mathrm{P}$-labeled substrates used in the reaction were $30 \mathrm{nM}, 36$ $\mathrm{nM}$ and 1-3 nM, respectively. Reactions were incubated for $30 \mathrm{~min}$ (unless otherwise stated) at $37^{\circ} \mathrm{C}$ (or $47.5^{\circ} \mathrm{C}$ for the thermophilic AacCas $12 \mathrm{~b}$ ) and quenched with formamide loading buffer (final concentration $45 \%$ formamide and 15 mM EDTA, with trace amount of xylene cyanol and bromophenol blue) for $3 \mathrm{~min}$ at $90^{\circ} \mathrm{C}$. The substrates and products were resolved by $12 \%$ ureadenaturing PAGE gel and quantified with Amersham Typhoon (GE Healthcare). 
For substrate turnover studies, the pre-assembled Cas12a-crRNA or Cas12a-crRNAactivator (target ssDNA or dsDNA) were incubated at $37^{\circ} \mathrm{C}$ for $10 \mathrm{~min}$, and $30 \mathrm{nM}$ of the preassembled RNP were used for each reaction with various substrate concentrations at 15, 30, 45, and $60 \mathrm{nM}$, respectively.

Fluorophore quencher (FQ)-labeled reporter assays. LbCas12a-crRNA complexes were preassembled by incubating $200 \mathrm{nM}$ LbCpf1 with $250 \mathrm{nM}$ crRNA and $4 \mathrm{nM}$ activator (ssDNA, dsDNA or ssRNA) at $37^{\circ} \mathrm{C}$ for $30 \mathrm{~min}$. The reaction was initiated by diluting LbCas $12 \mathrm{a}$ complexes to a final concentration of $50 \mathrm{nM}$ LbCas12a : $62.5 \mathrm{nM}$ crRNA : $1 \mathrm{nM}$ activator in a solution containing $1 \times$ Binding Buffer $(20 \mathrm{mM}$ Tris- $\mathrm{HCl}$, pH 7.5, $100 \mathrm{mM} \mathrm{KCl,} 5 \mathrm{mM} \mathrm{MgCl}, 1$ mM DTT, 5\% glycerol, $50 \mu \mathrm{g} \mathrm{ml}^{-1}$ heparin) and $50 \mathrm{nM}$ DNaseAlert substrate ${ }^{\mathrm{TM}}$ (IDT) or custom ssDNA/ssRNA FQ reporter substrates (Table S1). Reactions were incubated in a fluorescence plate reader (Tecan Infinite Pro F200) for up to 120 minutes at $37^{\circ} \mathrm{C}$ with fluorescence measurements taken every 30 seconds (DNaseAlert substrate $=\lambda$ ex: $535 \mathrm{~nm} ; \lambda$ em: $595 \mathrm{~nm}$, custom ssDNA/ssRNA FQ substrates $=\lambda \mathrm{ex}: 485 \mathrm{~nm} ; \lambda \mathrm{em}: 535 \mathrm{~nm}$ ).

For trans-cleavage rate determination, background-corrected fluorescence values were calculated by subtracting fluorescence values obtained from reactions carried out in the absence of target plasmid. The resulting data were fit to a single exponential decay curve (GraphPad Software), according to the following equation: Fraction cleaved $=\mathrm{A} \times(1-\exp (-\mathrm{k} \times \mathrm{t}))$, where $\mathrm{A}$ is the amplitude of the curve, $\mathrm{k}$ is the first-order rate constant, and $\mathrm{t}$ is time.

For Michaelis-Menten analysis, LbCas12a-crRNA-activator (target ssDNA or dsDNA) complexes were prepared as described above, and reaction was initiated by diluting LbCas12a complexes to a final concentration of $5 \mathrm{nM}$ LbCas12a : $6.25 \mathrm{nM}$ crRNA : $0.1 \mathrm{nM}$ activator (effective complex $=0.1 \mathrm{nM}$ ) in a solution containing $1 \times$ Binding Buffer and 0.001, 0.01, 0.1, 
$0.2,0.5,1$ or $2 \mathrm{uM}$ of DNaseAlert ${ }^{\mathrm{TM}}$ substrate (IDT). Reactions were incubated in a fluorescence plate reader for up to 30 minutes at $37^{\circ} \mathrm{C}$ with fluorescence measurements taken every 30 seconds $(\lambda$ ex: $535 \mathrm{~nm}$; $\lambda$ em: $595 \mathrm{~nm})$. The initial velocity $\left(\mathrm{V}_{0}\right)$ was calculated by fitting to a linear regression and plotted against the substrate concentration to determine the MichaelisMenten constants (GraphPad Software), according to the following equation: $\mathrm{Y}=\left(\mathrm{V}_{\max } \times\right.$ $\mathrm{X}) /\left(K_{\mathrm{m}}+\mathrm{X}\right)$, where $\mathrm{X}$ is the substrate concentration and $\mathrm{Y}$ is the enzyme velocity. The turnover number $\left(k_{\mathrm{cat}}\right)$ was determined by the following equation: $k_{\mathrm{cat}}=\mathrm{V}_{\max } / \mathrm{E}_{\mathrm{t}}$, where $\mathrm{E}_{\mathrm{t}}=0.1 \mathrm{nM}$.

HPV detection assays were performed as above, with the following modifications:

LbCas12a was pre-assembled with an HPV16 or HPV18-targeting crRNA and diluted in a solution containing $1 \times$ Binding Buffer, $50 \mathrm{nM}$ DNaseAlert substrate ${ }^{\mathrm{TM}}$ (IDT) and 1, 10, 100, or $1000 \mathrm{nM}$ of HPV16- or HPV18-containing plasmids. Reactions were incubated in a fluorescence plate reader for 60 minutes at $37^{\circ} \mathrm{C}$ with fluorescence measurements taken every 30 seconds ( $\lambda$ ex: $535 \mathrm{~nm}$; $\lambda$ em: $595 \mathrm{~nm})$. 


\section{Supplementary Figures S1-S8}

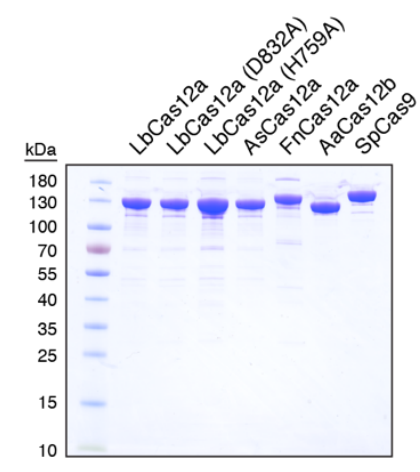

Fig. $\mathbf{S 1}$

Purification of Cas12 and Cas9 proteins. SDS-PAGE gel of all purified Cas 12 and Cas9 proteins used in this study. 

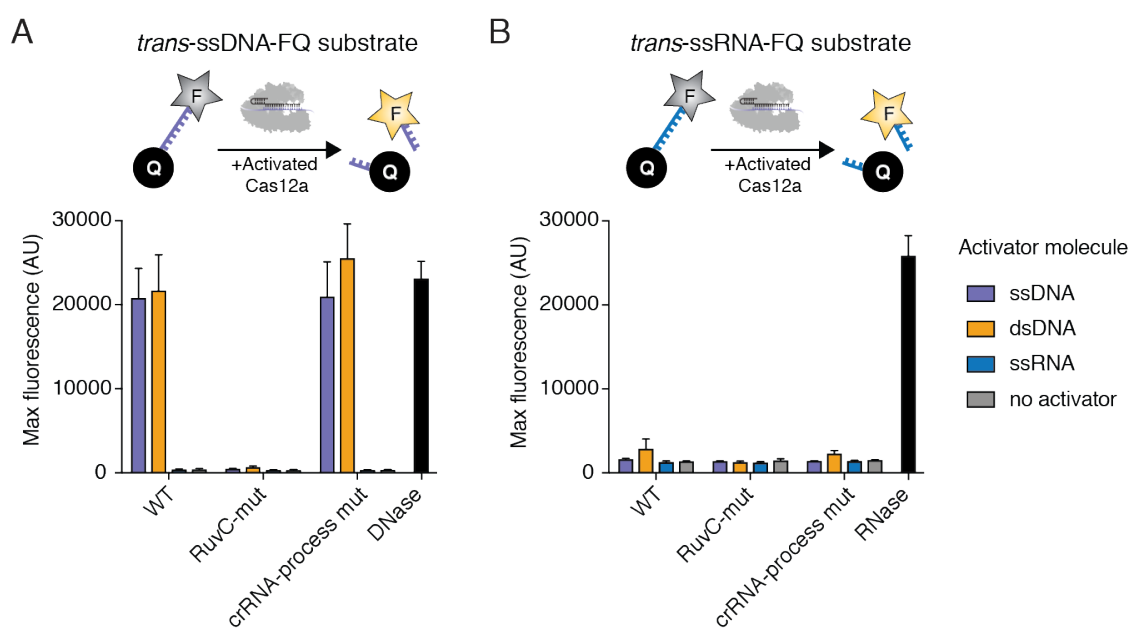

Fig. S2

LbCas12a is a DNA-activated general DNase. Quantification of maximum fluorescence signal generated after incubating LbCas12a-crRNA-activator with a custom (A) trans-ssDNA-FQ or (B) trans-ssRNA-FQ reporter for $1 \mathrm{~h}$ at $37^{\circ} \mathrm{C}$, with DNase I or RNase A controls where indicated. Error bars represent the mean \pm s.d., where $n=3$. 
A

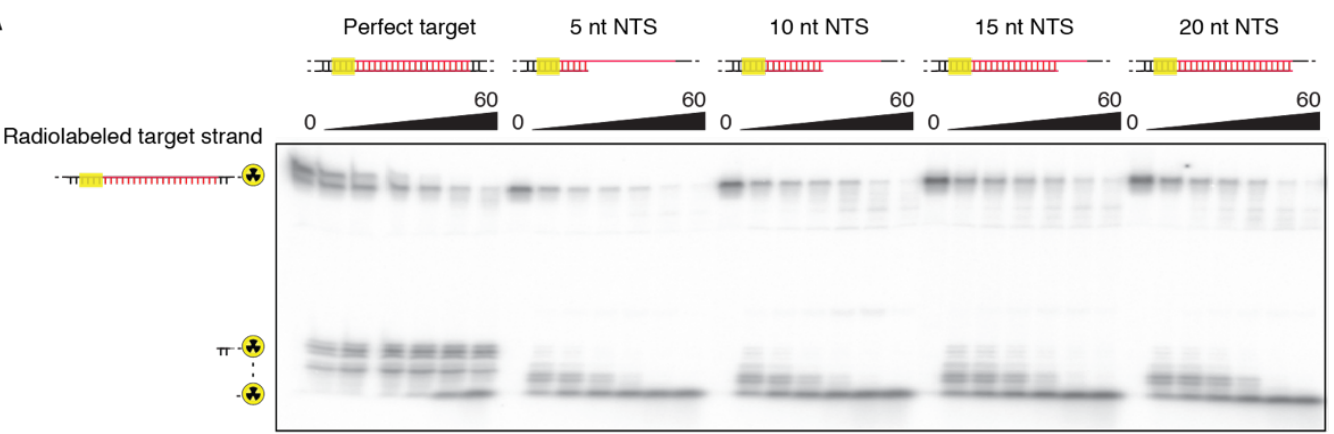

B
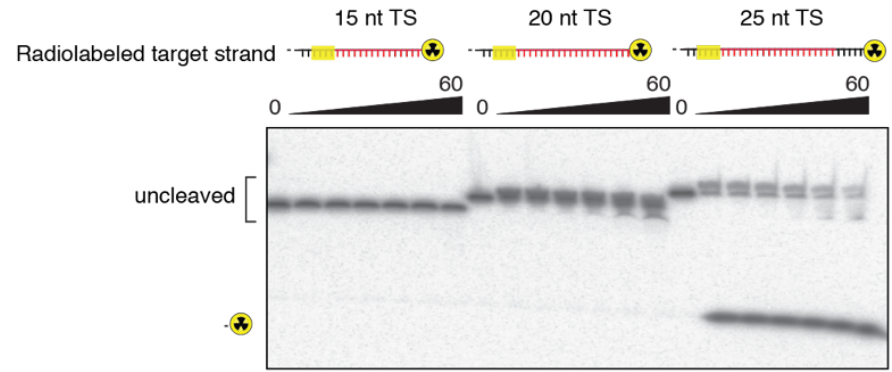

C
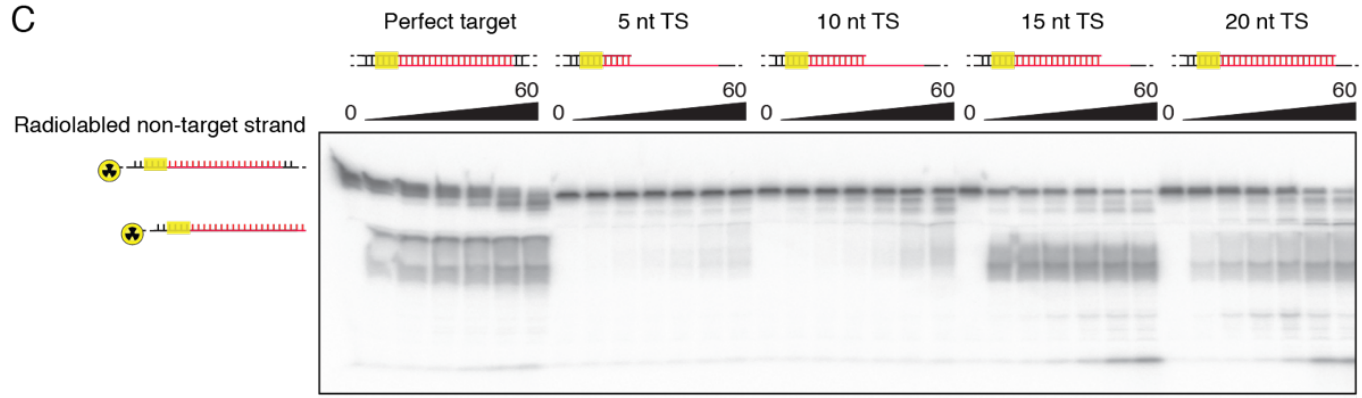

Fig. S3

Target strand recognition is a pre-requisite for single-stranded DNA cleavage. Cleavage timecourse assays using LbCas12a with (A) truncated target strand (TS) annealed to a radiolabeled non-target strand (NTS), (B) truncated and radiolabeled TS only, and (C) truncated NTS annealed to a radiolabeled TS. Timecourses represent minutes and cleavage products are resolved by denaturing PAGE. 


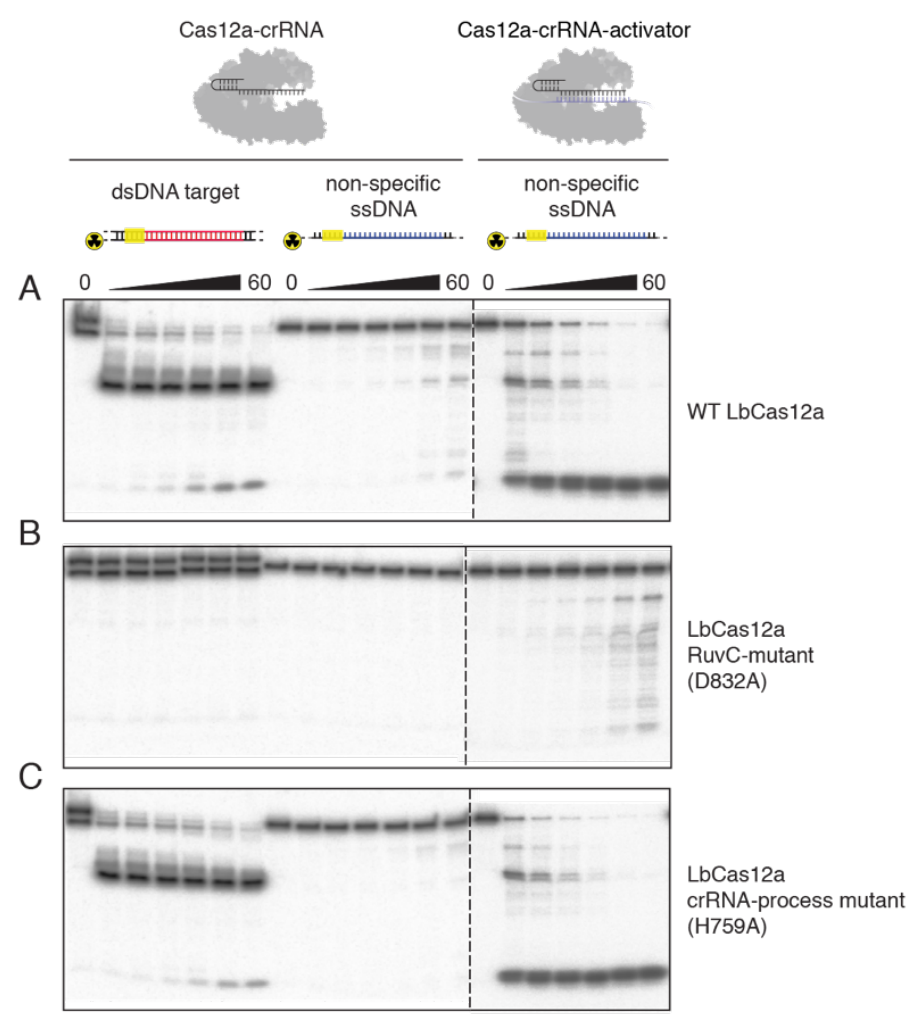

Fig. S4

The RuvC nuclease is responsible for activator-dependent, non-specific DNase activity.

Cleavage timecourse gel with radiolabeled target dsDNA and and non-specific ssDNA subsrates using (A) WT LbCas12a, (B) RuvC catalytic mutant (D832A) and (C) crRNA-processing mutant (H759A), with or without a ssDNA activator. Timecourses represent minutes and leavage products are resolved by denaturing PAGE. 


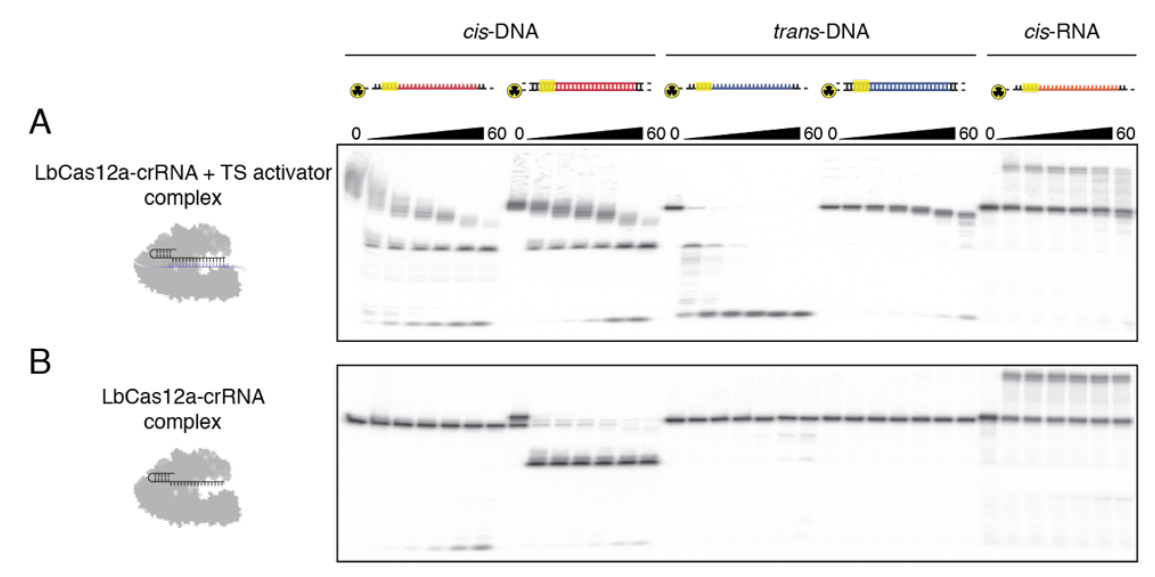

\section{Fig. S5}

\section{LbCas12a trans-cleavage degrades complementary and non-specific ssDNA, but not}

ssRNA. Cleavage timecourse gels of LbCas12a-crRNA complexes using (A) a ssDNA activator or (B) no activator with indicated radiolabeled substrates where cis indicates a complementary target and trans indicates a non-complementary sequence. Timecourses represent minutes and cleavage products are resolved by denaturing PAGE. 

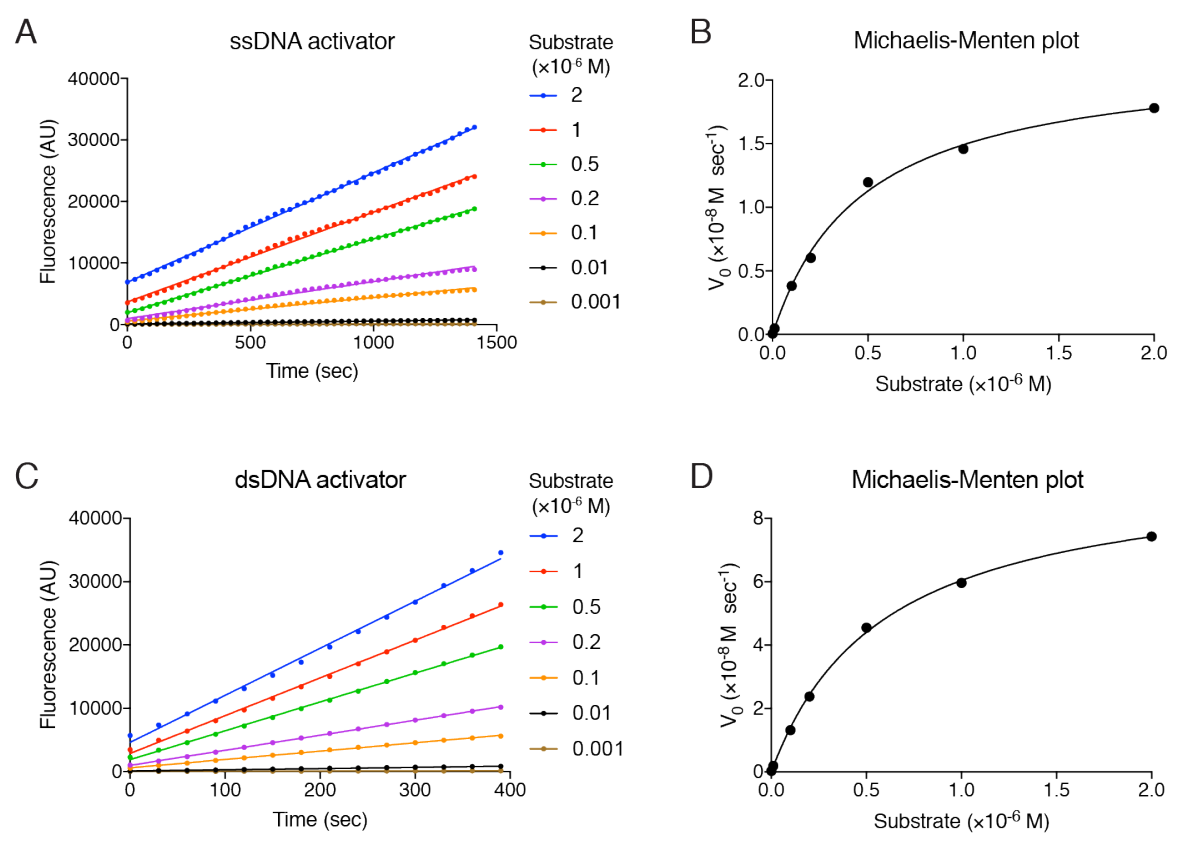

\begin{tabular}{|c|c|c|c|}
\hline Activator & $k_{\text {cat }}\left(\mathrm{sec}^{-1}\right)$ & $K_{\mathrm{m}}\left(\mathrm{M}^{-1}\right)$ & $k_{\mathrm{cat}} / K_{\mathrm{m}}\left(\mathrm{sec}^{-1} \mathrm{M}^{-1}\right)$ \\
\hline ssDNA & $2.50 \pm 0.30 \times 10^{2}$ & $4.94 \pm 0.16 \times 10^{7}$ & $5.10 \pm 0.47 \times 10^{8}$ \\
\hline dsDNA & $1.25 \pm 0.27 \times 10^{3}$ & $7.25 \pm 1.74 \times 10^{-7}$ & $1.73 \pm 0.18 \times 10^{9}$ \\
\hline
\end{tabular}

Fig. S6

\section{Michaelis-Menten analysis reveals robust trans-cleavage activity with a ssDNA and dsDNA}

activator. Representative plots of initial velocity versus time for a (A) ssDNA or (C) dsDNA activator, using $0.1 \mathrm{nM}$ effective LbCas12a-crRNA-activator complex and increasing DNaseAlert substrate concentrations at $37^{\circ} \mathrm{C}$. Michaelis-Menten fits for the corresponding (B) ssDNA or (D) dsDNA activator. (E) Calculated $k_{\text {cat }}, K_{\mathrm{m}}$ and $k_{\text {cat }} / K_{\mathrm{m}}$ values report the mean \pm s.d., where $n=3$. 


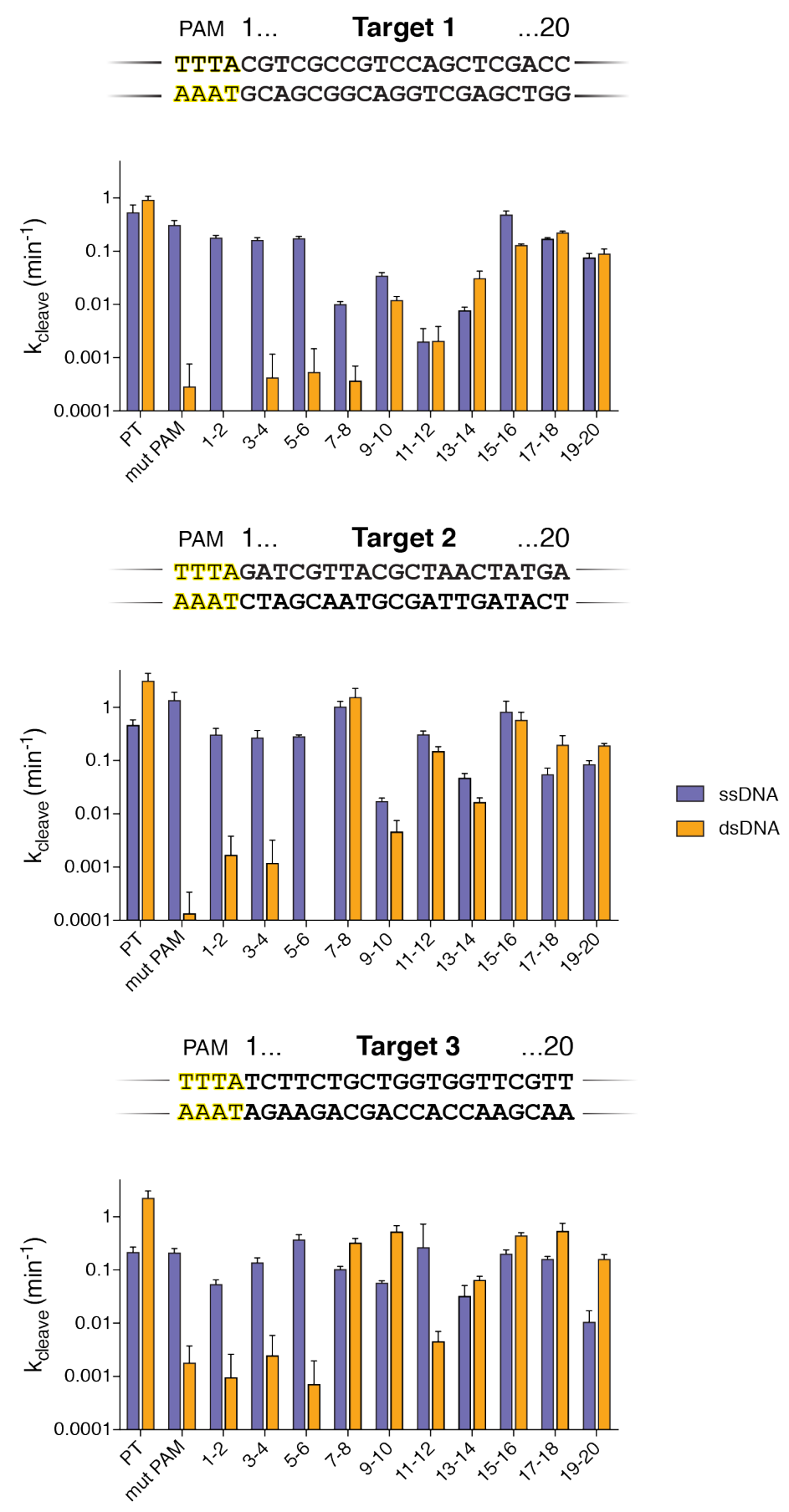

Fig. $\mathbf{S 7}$

The PAM sequence and PAM-proximal mismatches in a dsDNA activator provide specificity for trans-activation. Quantification of trans-cleavage kinetics using mismatched substrates for three distinct target sequences; error bars represent the mean \pm s.d., where $n=3$. 

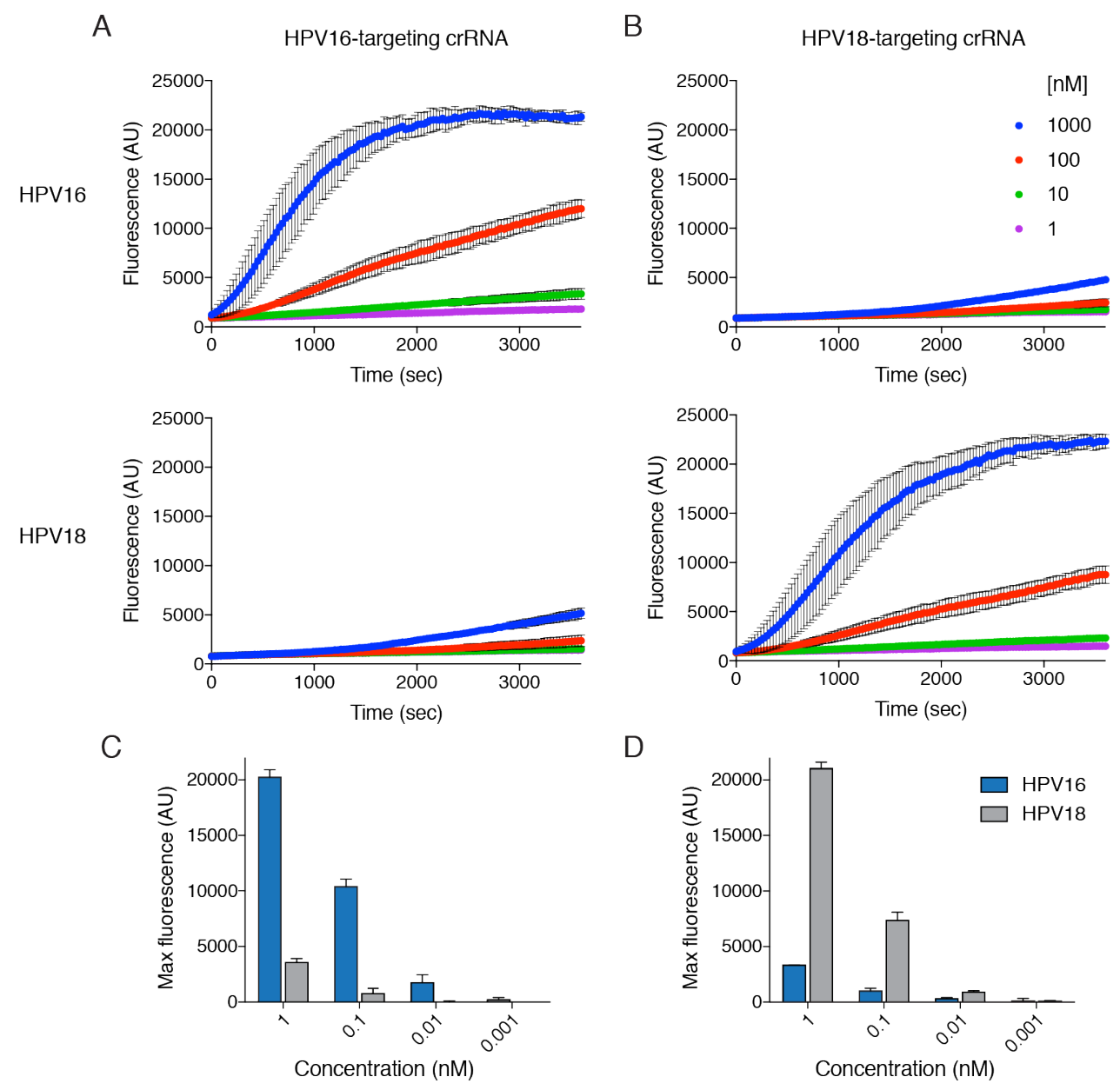

Fig. S8

Human papillomavirus (HPV) detection assay timecourse. Fluorescence timecourses with

LbCas12a preassembled with a crRNA targeting (A) HPV16 or (B) HPV16 in the presence of a dsDNA plasmid containing an HPV16 (top row) or HPV18 (middle row) genomic fragment and DNaseAlert substrate, with fluorescence measurements taken every 30 seconds for $1 \mathrm{~h}$ at $37^{\circ} \mathrm{C}$.

(C) Maximum fluorescence signal obtained from timecourses in (A) and (B). Error bars represent mean \pm s.d., where $n=3$. 
bioRxiv preprint doi: https://doi.org/10.1101/226993; this version posted November 29, 2017. The copyright holder for this preprint (which was not certified by peer review) is the author/funder. All rights reserved. No reuse allowed without permission.

\section{Supplementary Table S1}

\section{BNA}

LbCas12a crRNA - Target 1 UAAUUUCUACUAAGUGUAGAUCGUCGCCGUCCAGCUCGACC

LbCas12a crRNA - pUC19 UAAUUUCUACUAAGUGUAGAUCAACGUCGUGACUGGGAAAACCCU

LbCas12a crRNA - M13 UAAUUUCUACUAAGUGUAGAUAACGAACCACCAGCAGAAGA

LbCas12a crRNA - Target 2 UAAUUUCUACUAAGUGUAGAUGAUCGUUACGCUAACUAUGA

LbCas12a crRNA - Target 3 UAAUUUCUACUAAGUGUAGAUCCUGGGUGUUCCACAGCUGA

LbCas12a crRNA - HPV16 UAAUUUCUACUAAGUGUAGAUCUACAUUACAGGCUAACAAA

LbCas12a crRNA - HPV18 UAAUUUCUACUAAGUGUAGAUGUACAUUGCAAGAUACUAAA

AsCas12a crRNA - Target 2 UAAUUUCUACUCUUGUAGAUGAUCGUUACGCUAACUAUGA

FnCas12a crRNA - Target 2 UAAUUUUCUACUGUUGUAGAUGAUCGUUACGCUAACUAUGA

AaCas12b crRNA - Target 2 GUCUAGAGGACAGAAUUUUUCAACGGGUGUGCCAAUGGCCACUUUCCAGGUGGCAAAGCCCGUUGAGCUUCUCAAAUCUGAGAAGUGGCACGAUCGUUACGCUAACUAUGA

SpCas9 sgRNA - Target 1 cGUCGCCGUCCAGCUCGACCGUUUUAGAGCUAUGCUGUUUUGGAAACAAAACAGCAUAGCAAGUUAAAAUAAGGCUAGUCCGUUAUCAACUUGAAAAAGUGGCACCGAGUCGGUGC SpCas9 sgRNA - M13 AACGAACCACCAGCAGAAGAGUUUUAGAGCUAUGCUGUUUUGGAAACAAAACAGCAUAGCAAGUUAAAAUAAGGCUAGUCCGUUAUCAACUUGAAAAAAGUGGCACCGAGUCGGUGC SpCas9 sgRNA - Target 2 GAUCGUUACGCUAACUAUGAGUUUUAGAGCUAUGCUGUUUUGGAAACAAAACAGCAUAGCAAGUUARAAUAAGGCUAGUCCGUUAUCAACUUGAAAAAGUGGCACCGAGUCGGUGC Target 1 ssRNA

Target 2 ssRNA GCCGGGGUGGUGCCCAUCCUGGUCGAGCUGGACGGCGACGUAAACGGCCACAAGC

Plasmids

M13mp18 ssDNA phage https://benchling.com/s/seq-mnJjAqQ9tHfSPbKTNIPT pUC19 dsDNA plasmid https://benchling.com/s/seq-x4orD9lwDx2iHa6Ns3pR HPV16-containing plasmid https://benchling.com/s/seq-g0t5PySBcx $4 \mathrm{cwnz} 2 \mathrm{~h} 82 \mathrm{~W}$ HPV18-containing plasmid https://benchling.com/s/seq-hv244BG5z200N23SUZwq

DNA

non-specific NTS AGCTTGTCTGCCATGGACATGCAGACTATACTGTTATTGTTGTACAGACCGAATTCCC

non-specific TS

Target 1_NTS

Target 1_TS

Target 1_20-19_NTS

Target 1_20-19_TS

Target 1_18-17_NTS

Target 1_18-17_TS

Target 1_16-15_NTS

Target 1_16-15_TS

Target 1_14-13_NTS

Target 1_14-13_TS

Target 1_12-11_NTS

Target 1_12-11_TS

Target 1_10-9_NTS

Target 1_10-9_TS

Target 1_8-7_NTS

Target 1_8-7_TS

Target 1_6-5_NTS

Target 1_6-5_TS

Target 1_4-3_NTS

Target 1_4-3_TS

Target 1_2-1_NTS

Target 1_2-1_TS

Target 1_mut-PAM_NTS

Target 1_mut-PAM_TS

Target 1_5nt_TS

Target 1_10nt_TS

Target 1_15nt_TS

Target 1_20nt_TS

Target 1_25nt_TS

Target 1_5nt_NTS

Target 1_10nt_NTS

Target 1_15nt_NTS

Target 1_20nt_NTS

GGGAATTCGGTCTGTACAACAATAACAGTATAGTCTGCATGTCCATGGCAGACAAGCT

GCTTGTGGCCGTTTACGTCGCCGTCCAGCTCGACCAGGATGGGCACCACCCCGGC GCCGGGGTGGTGCCCATCCT GGTCGAGCTGGACGGCGACGTAAACGGCCACAAGC GCTTGTGGCCGTTTACGTCGCCGTCCAGCTCGAGGAGGATGGGCACCACCCCGGC GCCGGGGTGGTGCCCATCCTCCTCGAGCTGGACGGCGACGTAAACGGCCACAAGC GCTTGTGGCCGTTIACGTCGCCGTCCAGCTCCTCCAGGATGGGCACCACCCCGGC GCCGGGGTGGTGCCCATCCTGGAGGAGCTGGACGGCGACGTAAACGGCCACAAGC GCTTGTGGCCGTTTACGTCGCCGTCCAGCAGGACCAGGATGGGCACCACCCCGGC GCCGGGGTGGTGCCCATCCTGGTCCTGCTGGACGGCGACGTAAACGGCCACAAGC GCTTGTGGCCGTTIACGTCGCCGTCCACGTCGACCAGGATGGGCACCACCCCGGC GCCGGGGTGGTGCCCATCCTGGTCGACGTGGACGGCGACGTAAACGGCCACAAGC GCTTGTGGCCGTT TACGTCGCCGTCGTGCTCGACCAGGATGGGCACCACCCCGGC GCCGGGGTGGTGCCCATCCT GGTCGAGCACGACGGCGACGTAAACGGCCACAAGC GCTTGTGGCCGTTTACGTCGCCGAGCAGCTCGACCAGGATGGGCACCACCCCGGC GCCGGGGTGGTGCCCATCCTGGTCGAGCTGCTCGGCGACGTAAACGGCACAAGC GCTTGTGGCCGTTTACGTCGCGCTCCAGCTCGACCAGGATGGGCACCACCCCGGC GCCGGGGTGGT GCCCATCCTGGTCGAGCTGGAGCGCGACGTAAACGGCCACAAGC GCTTGTGGCCGTTTACGTCCGCGTCCAGCTCGACCAGGATGGGCACCACCCCGGC GCCGGGGTGGTGCCCATCCTGGTCGAGCTGGACGCGGACGTAAACGGCCACAAGC GCTTGTGGCCGTTTACGAGGCCGTCCAGCTCGACCAGGATGGGCACCACCCCGGC GCCGGGGTGGTGCCCATCCTGGTCGAGCTGGACGGCCTCGTAAACGGCCACAAGC GCTTGTGGCCGTTTAGCTCGCCGTCCAGCTCGACCAGGATGGGCACCACCCCGGC GCCGGGGTGGTGCCCATCCT GGTCGAGCTGGACGGCGAGCTAAACGGCCACAAGC GCTTGTGGCCGAGCACGTCGCCGTCCAGCTCGACCAGGATGGGCACCACCCCGGC GCCGGGGTGGTGCCCATCCTGGTCGAGCTGGACGGCGACGTGCTCGGCCACAAGC

CGACGTAAACGGCCACAAGC GACGGCGACGTAAACGGCCACAAGC AGCTGGACGGCGACGTAAACGGCCACAAGC GGTCGAGCTGGACGGCGACGTAAACGGCCACAAGC ATCCTGGTCGAGCTGGACGGCGACGTAAACGGCACAAGC

GCTTGTGGCCGTTTACGTCG

GCTTGTGGCCGTTTACGTCGCCGTC

GCTTGTGGCCGTTIACGTCGCCGTCCAGCT

GCTTGTGGCCGTTTACGTCGCCGTCCAGCTCGACC 
bioRxiv preprint doi: https://doi.org/10.1101/226993; this version posted November 29, 2017. The copyright holder for this preprint (which was not certified by peer review) is the author/funder. All rights reserved. No reuse allowed without permission.

\begin{tabular}{|c|c|}
\hline & ITTAGATCGTTACGCTAACTATGAGGGCTGTCTGTGGAATGCTA \\
\hline Target 2_TS & AGCATTCCACAGACAGCCCTCATAGTTAGCGTAACGATCTAAAGTTTTGTCGTC \\
\hline Target 2_20-19_NTS & ACGACAAAACTTTAGATCGTTACGCTAACTATCTGGGCTGTCTGTGGAATGCTA \\
\hline arget 2_20-19_TS & TAGCATTCCACAGACAGCCCAGATAGTTAGCGTAACGATC TAAAGTTTTGTCGTC \\
\hline Target 2_18-17_NTS & GACGACAAAACTTTAGATCGTTACGCTAACTTAGAGGGCTGTCTGTGGAATGCTA \\
\hline Target 2_18-17_TS & TAGCATTCCACAGACAGCCCTCTAAGTTAGCGTAACGATCTAAAGTTTTGTCGTC \\
\hline Target 2_16-15_NTS & GACGACAAAACTTTAGATCGTTACGCTAAGAATGAGGGCTGTCTGTGGAATGCTA \\
\hline Target 2_16-15_TS & TAGCATTCCACAGACAGCCCTCATTCTTAGCGTAACGATCTAAAGTTTTGTCGTC \\
\hline Target 2_14-13_NTS & GACGACAAAACTTTAGATCGTTACGCTTTCTATGAGGGCTGTCTGTGGAATGCTA \\
\hline Target 2_14-13_TS & TAGCATTCCACAGACAGCCCTCATAGAAAGCGTAACGATCTAAAGTTTTGTCGTC \\
\hline Target 2_12-11_NTS & GACGACAAAACTTTAGATCGTTACGGAAACTATGAGGGCTGTCTGTGGAATGCTA \\
\hline Target 2_12-11_TS & AGCATTCCACAGACAGCCCTCATAGTTTCCGTAACGATCTAAAGTTTTGTCGTC \\
\hline$t 2$ & GACGACAAAACTTTAGATCGTTAGCCTAACTATGAGGGCTGTCTGTGGAATGCTA \\
\hline et 2_10 & TAGCATTCCACAGACAGCCCTCATAGTTAGGCTAACGATCTAAAGTTTTGTCGTC \\
\hline et 2_8-7_ & GACGACAAAACTTTAGATCGTATCGCTAACTATGAGGGCTGTCTGTGGAATGCTA \\
\hline Target 2_8-7_TS & ГAGCATTCCACAGACAGCCCTCATAGTTAGCGATACGATCTAAAGTTTTGTCGTC \\
\hline Target 2_6-5_NTS & GACGACAAAACTTTAGATCCATACGCTAACTATGAGGGCTGTCTGTGGAATGCTA \\
\hline Target 2_6-5_TS & TAGCATTCCACAGACAGCCCTCATAGTTAGCGTATGGATCTAAAGTTTTGTCGTC \\
\hline Target 2_4-3_NTS & GACGACAAAACTTTAGAAGGTTACGCTAACTATGAGGGCTGTCTGTGGAATGCTA \\
\hline Target 2_4-3_TS & TAGCATTCCACAGACAGCCCTCATAGTTAGCGTAACCTTCTAAAGTTTTGTCGTC \\
\hline Target 2_2-1_NTS & GACGACAAAACTTTACTTCGTTACGCTAACTATGAGGGTGTCTGTGGAATGCTA \\
\hline Target 2_2-1_TS & TAGCATTCCACAGACAGCCCTCATAGTTAGCGTAACGAAGTAAAGTTTTGTCGTC \\
\hline Target 2_mut- & GACGACAAAACAGCAGATCGTTACGCTAACTATGAGGGCTGTCTGTGGAATGCTA \\
\hline Tars & TAGCATTCCACAGACAGCCCTCATAGTTAGCGTAACGATCTGCTGTTTTGTCGTC \\
\hline & GTTGTGTTAGTTTACCTGGGTGTTCCACAGCTGATAGTGATTGCCTTGAATAAA \\
\hline Target 3_TS & TTTATTCAAGGCAATCACTATCAGCTGTGGAACACCCAGGTAAACTAACACAACT \\
\hline Target 3_20-19_NTS & AGTTGTGTTAGTTTACCTGGGTGTTCCACAGCTCTTAGTGATTGCCTTGAATAAA \\
\hline Target 3_20 & TTTATTCAAGGCAATCACTAAGAGCTGTGGAACACCCAGGTAAACTAACACAACT \\
\hline NTS & AGTTGTGTTAGTTTACCTGGGTGTTCCACAGGAGATAGTGATTGCCTTGAATAAA \\
\hline Targ & TTTATTCAAGGCAATCACTATCTCCTGTGGAACACCCAGGTAAACTAACACAACT \\
\hline NTS & AGTTGTGTTAGTTTACCTGGGTGTTCCACTCCTGATAGTGATTGCCTIGAATAAA \\
\hline Tars & TTTATTCAAGGCAATCACTATCAGGAGTGGAACACCCAGGTAAACTAACACAACT \\
\hline Tar & AGTTGTGTTAGTTTACCTGGGTGTTCCTGAGCTGATAGTGATTGCCTTGAATAAA \\
\hline t 3_1 & TTTATTCAAGGCAATCACTATCAGCTCAGGAACACCCAGGTAAACTAACACAACT \\
\hline Tar & GTTGTGTTAGTTIACCTGGGTGTTGGACAGCTGATAGTGATTGCCTTGAATAAA \\
\hline Tar & АТТАСТАTCAGCTGTCCAACACCCAGGTAAACTAACACAACT \\
\hline Tar & AGTTGGTTAGTTTACCTGGGTGAACCACAGCTGATAGTGATTGCCTTGAATAAA \\
\hline 3 & TTTATTCAAGGCAATCACTATCAGCTGTGGTTCACCCAGGTAAACTAACACAACT \\
\hline 3_8 & AGTTGTGTTAGTTTACCTGGGACTTCCACAGCTGATAGTGATTGCCTTGAATAAA \\
\hline Target 3_8-7_TS & TTTATTCAAGGCAATCACTATCAGCTGTGGAAGTCCCAGGTAAACTAACACAACT \\
\hline Target 3_6-5_NTS & AGTTGTGTTAGTTTACCTGCCTGTTCCACAGCTGATAGTGATTGCCTTGAATAAA \\
\hline Target 3_6-5_TS & TTTATTCAAGGCAATCACTATCAGCTGTGGAACAGGCAGGTAAACTAACACAACT \\
\hline et 3_4-3_N & AGTTGTGTTAGTTTACCACGTTTTCCACAGCTGATAGTGATTGCCTTGAATAAA \\
\hline t3_4 & TTTATTCAAGGCAATCACTATCAGCTGTGGAACACCGTGGTAAACTAACACAACT \\
\hline Tar & AGTTGTGTTAGTTTAGGTGGGTGTTCCACAGCTGATAGTGATTGCCTTGAATAAA \\
\hline la & ATCACTATCAGCTGTGGAACACCCACCTAAACTAACACAACT \\
\hline 3_r & AGTTGTGTTAGAGCACCTGGGTGTTCCACAGCTGATAGTGATTGCCTTGAATAAA \\
\hline Target 3_mu & TTATTCAAGGCAATCACTATCAGCTGTGGAACACCCAGGTGCTCTAACACAACT \\
\hline \multicolumn{2}{|l|}{ ubstrate } \\
\hline & /56-FAM/ \\
\hline IAT & /56-FAM/rUrUrArUrU/ \\
\hline Vase & roprietary \\
\hline
\end{tabular}

Table S1

Nucleic acids used in this study.

\section{References:}

1. M. Jinek et al., Structures of Cas9 endonucleases reveal RNA-mediated conformational activation. Science 343, 1247997 (2014). 